\title{
Research on Application of Chinese Traditional Patterns in Soft Decorative Design of Hotel Space
}

\author{
Tingting Wang \\ Qingdao Vocational and Technical College of Hotel Management, Qingdao, China
}

Keywords: Application, Chinese Traditional Patterns, Soft Decorative Design, Hotel Space

\begin{abstract}
Chinese traditional pattern has a long history, it is very artistic appeal and it is the crystallization of human labor wisdom and the product of people's thinking of different times. In this paper, the application of the traditional pattern design in the soft design of modern hotels in China is discussed, and the development status and problems of the traditional patterns in the hotel soft-installation design are analyzed. Then, some methods of soft decoration design are proposed, hoping bring a better impact on the hotel soft decorative design.
\end{abstract}

\section{Introduction}

Today, a variety of hotel space after another, highlighting the performance of individual characteristics, the cultural atmosphere and the hotel space environment corresponding to express the theme of space environment, comfort, elegance, tranquility and many other civilizations and cultural deep meaning, gives the feeling of beauty, Pleasant customer mood. This reflects the people living in the material level of rising conditions, began to have a new spiritual life requirements, that is, in this hotel space environment with the formation of emotional echo. Hotel space environment for each successful example must have a unique style, to reflect their own spiritual and cultural outlook, so that customers impressed, such as Chinese South Beauty, Little Sheep and other hotel chains. Therefore, the unique style and personality is the hotel space environment, the central idea and soul.

\section{The Development Status Quo of the Traditional Pattern}

Modern hotel decoration design attaches great importance to the use of traditional Chinese patterns to carry out innovative decorative design and this can enrich the cultural connotation of the hotel, adding to the decoration of the Chinese elements in the hotel. The use of traditional Chinese patterns of soft-loaded design, the hotel will bring a unique beauty, enhance the hotel's luxury.

Modern hotel in the soft-loaded design, both show the hotel decorative features, and let the hotel's artistic atmosphere is improved, making modern hotel software reflects the contemporary socio-economic level and technological level, but also reflects the modern society Cultural ideology, regional folk customs and aesthetic point of view. The soft design of traditional patterns is the embodiment of the era of decorative culture, is the dissemination of traditional Chinese culture is a good way. Modern hotel in the soft design of the full application of the traditional pattern elements, making the modern hotel fully demonstrates the contemporary social and cultural connotations, some hotels in the decorative design will be the essence of Western modern design and traditional Chinese patterns combined pattern, reflects the Good innovative design spirit, which carries forward and inheritance of traditional Chinese decorative pattern is a big challenge.

Through the soft decoration design in modern hotels, to meet customer service requirements, while enhancing the hotel's social awareness and improve the customer and the community on the hotel's evaluation, which brings good economic benefits for the hotel. In recent years, the design and application of the traditional pattern design in Chinese hotel soft-fitting design is novel and profound, which fully promotes the level of soft-installation design of hotels in China. However, in a large number of Chinese traditional pattern design of repeated applications, coupled with the lack of innovation in the application of some materials, making Chinese modern hotel in the soft-loaded design there are some problems. 


\section{The Development of Traditional Patterns}

The Abuse and Blind Imitation Plagiarism of Pattern Soft Design are Serious. At present, many hotels in China have some problems in the design of Chinese traditional patterns, such as the old pattern, the repeated use of materials, the lack of regional cultural characteristics, blind imitation and other phenomena. As the soft-installed designers do not have a deep understanding of the soft-loaded cultural design, making many hotels can not accurately locate the market, bringing a soft decorative design of light decoration features, reducing the hotel's service functions. There are many soft-installed designers do not understand the study of regional culture in the design of foreign software design is only a simple imitation and plagiarism, making the hotel soft-fitted design lacks the hotel's own vitality, a lot of soft-fitted design star hotel In the decorative culture of the abuse of the lack of geographical characteristics, making it difficult to integrate into the soft-loaded design of the hotel's own characteristics.

Chinese Soft Decorative Design Industry Is Not Mature. The development of soft hotel industry, there is still much room for improvement, a lot of soft-installed designers are lack of understanding of the status quo of soft-fitted design, they can not be very good use of traditional Chinese patterns for soft-loaded design, resulting in a lot of hotels Soft-loaded style, material are surprisingly similar, a serious impediment to the development of Chinese hotel soft-installed design. Different areas of the hotel should be on their own soft-loaded design have different requirements and style, because the regional cultural characteristics are different, so the hotel's soft equipment should have the characteristics of the region or the hotel's unique taste, to create a distinctive hotel Image.

Ignore The Spiritual Culture of Soft-Loaded Hotel Design. Currently in the hotel decoration industry, many designers are through the decoration style and form of the transformation to the hotel soft design, and not the hotel's own characteristics and the spirit of the connotation of the link design, making the hotel soft design lack of cultural spirit Connotation. These years, Chinese hotel design is very focused on mining and improves the hotel culture and its connotation, with particular emphasis on traditional culture and geographical and cultural characteristics of the excavation, and in some hotel software design has achieved good results. Designers will be some unique cultural characteristics into the hotel's soft decoration products to give soft decoration products a new cultural significance and meaning, making the hotel has been its customers and the community of higher praise. Modern hotel as a public service venues, to meet the guests accommodation, dining, conference, entertainment and other needs, but also through the hotel's own furnishings, such as the design of soft ornaments to give guests spiritual and cultural aspects of the infection, For guests to enjoy the spirit of pleasure and enjoyment.

The Challenge of the Chinese Traditional Pattern in the Soft Decorate Design of the Hotel under the Multi-Culture. With the development of economic globalization, international hotels began to expand the Chinese market, such as Sheraton, Hilton, Starwood, etc., they have the world's advanced hotel soft design concept. Although the international famous hotel to enter the Chinese market, to the domestic hotel industry has brought great challenges, but these hotels advanced soft design concept gives us a good reference, we need to face competitors, learn from, To learn the essence of its design and design, the traditional pattern in the hotel soft-loaded design cleverly reflected, carry forward our traditional culture, leading the soft decoration design industry continue to forge ahead.

\section{The Soft Decorative Design Principles of Hotel Space}

The Space Design Must Meet the Functional Requirements. Practicality is the basic principle of the hotel space environment design, but also the primary condition, only as a fundamental to practice in life. Therefore, the design of the rationality of the hotel space environment is the form, size, space division of the prerequisites in the channel and dining table layout and reasonable circumstances, dining and meals convenient and quick, so as to meet the needs of the hotel. At the same time, but also from the visual comfort of customers, from the spatial interface landscape, 
furnishings, decoration and other aspects of the design requirements. Here are two different functions of the hotel space to illustrate. The main flavor of the restaurant is to highlight the flavor characteristics, such as the Chinese cuisine in Zhejiang cuisine, Fujian, Hunan and other international cuisine with Thai, Korean and so on. Bar as a leisure-oriented hotel environment, the main style is casual, comfortable.

The Space Design Must Meet the Engineering and Technical Requirements. In the design of hotel space environment, engineering technology exists as a rigid condition in the whole design concept. The material means to isolate each space is structure and material, and it has certain necessity. The design must obey the requirements of structure and material. Light, sound, heat, etc. is to create a comfortable, comfortable atmosphere of the physical means, the above facilities in the hotel space design which need to set aside space, and to meet the technical requirements.

The Hotel Space Has a Variety of Forms of Composition. It's hard to imagine dining in a restaurant that's evenly tidy but unprocessed. This space gives a sense of monotony. In this same space, the use of physical separation or surrounded by the use of partition in the form of this monotonous space disruption reorganization, making it a number of different forms of space, then give the space added a lot of fun, and Conducive to people's exchanges, giving a sense of pleasure. This shows that people are full of colorful and interesting space full of desire. Therefore, in the design which should create a multi-type space environment as a prerequisite for the small space of originality and finally integrated into a perfect combination.

\section{The Embodiment and Development Trends of Traditional Chinese Patterns in the Hotel Space}

For the choice of table seating, customers tend to avoid large flow of people or near-door locations. This is because these sites have a certain degree of interference on communication and communication, the lack of privacy protection, which includes the space to block the sound and sight, etc. requirements. Therefore, in the dining environment and more set "end", such as against the wall deck, so that customers "end" of the psychological has been met. By enclosing the table in a separate space, the shelter space minimizes interference, gives people a sense of security, maintains a certain distance from the outside world, and blocks the embarrassment of others' peeps , While avoiding the dorsal side was crowded with the sense of crowded, this seat favored. Nowadays, the design of space and space is more and more used in life, so that the space can provide a stable atmosphere, and give people a sense of security. In the design of the division of space, the border of the space environment should try to use vertical entities to implement enclosure, as far as possible to ensure that each side of the table is more than a vertical entity. The banquet hall is a special case, which is to achieve the participation of all participants in the exchange of places, the layout of the purpose of the table so that these people communication, entertainment, no privacy, to create a warm and good atmosphere to break the boundary of isolated space, this When they need to table evenly arranged, four facing empty. Different interactions require different interpersonal distances to meet. For example, some of the hotel layout to give people a sense of spacious and bright; some hotel layout is divided into large space in a few small space, people can not only enjoy the comfort of small space, but also feel the overall atmosphere of large space; Some of the hotel layout is the use of partition will table divided into a separate area, with privacy and can make people feel the overall atmosphere; some hotel layout of the inter-seat, suitable for small private parties, business negotiations.

In short, the design of spatial distance according to interpersonal distance appropriate design, gender, ethnic, environmental and interpersonal distance, so the design of space also need to local conditions. The layout of the dining table based on space, to meet the local cultural and psychological needs of consumers, while ensuring the diversity of choices is the soul of the hotel space design ideas.

Has been high-end consumer space is the place where the hotel space environment, so the identity status and luxury space design has become a trend, as a symbol of wealth in the design of them. High-grade materials, a large number of stacked design style ignores the needs of customers, along with the progress of the times, the Chinese design will replace this impetuous. The concept of 
people-oriented doomed to design to serve the people, the hotel space environment design perfect and reasonable, is the concept of the pursuit of the designer, the pursuit of new differences as a basis for treatment, abandon the past extravagance and trivial to highlight the luxury of cultural essence Through the intimate design language to show a unique personality. Service as a standard feature of the hotel space environment to achieve a closed to open to the transition from a comprehensive to individual development, decoration layout from the single to the diversification of evolution, from the initial focus on social benefits to focus on the economy Efficiency development, so that the management of services to the development of Chinese services. The combination of natural environment and man-made environment is a new concept of hotel space environment design and that is to achieve the common development between the two, with green design as the new direction of advocacy. The biosphere and the non-renewable resources in the ecosystem, the use of renewable resources to build a recyclable system, and actively use solar energy and light energy, change the passive way of artificial ventilation, resource conservation, green, to achieve a true sense of natural resources Protection and utilization of environmentally friendly decorative materials will be fully applied to the design of them.

\section{Conclusion}

With the continuous progress of society and the stable development of economy, people's aesthetic level is also increasing. In a sense, the Chinese traditional patterns in the hotel space environment reflect a flavor of the times, so the use of Chinese traditional patterns in the hotel space design is the trend of hotel environment design.

\section{References}

[1] Huifang Zhou: Building knowledge, Vol. 6 (2004) No 53, p.25-26

[2] Hongli Zhang: Young Writer, Vol. 12 (2005) No 27, p.74-76

[3] Qin Guo: Fujian Architecture and Construction, Vol. 1 (2006) No 33, p.11-14

[4] Jieming Liu: Guangxi Normal University, Vol. 3 (2007) No33, p.121-124

[5] Wang Hui: Chinese Architecture, Vol. 1 (2012) No 33, p.52-60 